\title{
MPSA effects on copper electrodeposition investigated by molecular dynamics simulations
}

John N. Harb

john_harb@byu.edu

Richard L. Rowley

rowley@byu.edu

Dean R. Wheeler

dean_wheeler@byu.edu

Clint G. Guymon

Follow this and additional works at: https://scholarsarchive.byu.edu/facpub

Part of the Chemical Engineering Commons

\section{Original Publication Citation}

Guymon, Clint G., John N. Harb, Richard L. Rowley, and Dean R. Wheeler. "MPSA effects on copper electrodeposition investigated by molecular dynamics simulations." The Journal of Chemical Physics 128 (28)

\section{BYU ScholarsArchive Citation}

Harb, John N.; Rowley, Richard L.; Wheeler, Dean R.; and Guymon, Clint G., "MPSA effects on copper electrodeposition investigated by molecular dynamics simulations" (2008). Faculty Publications. 211. https://scholarsarchive.byu.edu/facpub/211 accepted for inclusion in Faculty Publications by an authorized administrator of BYU ScholarsArchive. For more information, please contact ellen_amatangelo@byu.edu. 


\title{
MPSA effects on copper electrodeposition investigated by molecular dynamics simulations
}

\author{
Clint G. Guymon, ${ }^{\text {a) }}$ John N. Harb, Richard L. Rowley, and Dean R. Wheeler ${ }^{\text {b) }}$ \\ Department of Chemical Engineering, Brigham Young University, Provo, Utah 84602, USA
}

(Received 21 December 2006; accepted 16 November 2007; published online 31 January 2008)

\begin{abstract}
In superconformal filling of copper-chip interconnects, organic additives are used to fill high-aspect-ratio trenches or vias from the bottom up. In this study we report on the development of intermolecular potentials and use molecular dynamics simulations to provide insight into the molecular function of an organic additive (3-mercaptopropanesulfonic acid or MPSA) important in superconformal electrodeposition. We also investigate how the presence of sodium chloride affects the surface adsorption and surface action of MPSA as well as the charge distribution in the system. We find that $\mathrm{NaCl}$ addition decreases the adsorption strength of MPSA at a simulated copper surface and attenuates the copper-ion association with MPSA. The model also was used to simulate induced-charge effects and adsorption on a nonplanar electrode surface. () 2008 American Institute of Physics. [DOI: 10.1063/1.2824928]
\end{abstract}

\section{INTRODUCTION}

In 1989, IBM showed that damascene copper plating can be used to fill microsized trenches or vias that electrically connect devices on an integrated circuit. In 1997, the first microprocessor using damascene plating technology was produced. This represented a significant advancement in the microelectronics industry as copper is more resistant to electromigration (a problem that leads to device failure) and more conductive than aluminum, the previously used material.

In the damascene process, copper is electrolytically deposited in the presence of organic additives in the plating bath. The appropriate additives enable the copper deposit to fill high-aspect-ratio trenches and vias bottom up and without voids. It has been postulated that such "superconformal" filling results from accumulation of a catalytic or accelerator species upon concave areas of the metal surface. ${ }^{1,2}$ The increased accelerator surface concentration results in an increased deposition rate at the bottom of the concave feature and the subsequent formation of the observed convex deposit over the fully filled feature. Another hypothesis is that the bottom of the convex feature is less accessible to species that inhibit deposition due to diffusional resistance. ${ }^{3}$ While either of these theories can account for macroscopic observations of deposit growth, additional information is required to further understand the process on an atomic scale.

Multiple additives labeled as accelerators, suppressors, and levelers are used to generate high-quality interconnects in the damascene process. Bis(sodiumsulfopropyl) disulfide (SPS) in the presence of small quantities of chloride ion is considered an accelerator. SPS is essentially a dimer of 3-mercaptopropanesulfonic acid (MPSA). There is evidence that complexes of MPSA with cuprous ion in the presence of

\footnotetext{
a)Also at Safety Management Services Inc., West Jordan, UT 84088.

${ }^{b)}$ Author to whom correspondence should be addressed. Electronic mail: wheeler@et.byu.edu.
}

chloride may be the source of the observed acceleration of copper deposition. ${ }^{4,5}$ Superfilling baths with added MPSA and chloride ions have been shown to accelerate copper electrodeposition. ${ }^{6-8}$

Although SPS or MPSA are labeled as accelerators, their action is more complex than this. In additive baths where the composition of chloride ion is small $(<1 \mathrm{ppm})$, SPS or MPSA actually inhibits the rate of copper electrodeposition. ${ }^{5,8}$ When chloride is subsequently added, the rate is accelerated above the initial additive-free value. Some have attributed the acceleration with chloride ion to copper-chloride complexation near the sulfonate group., ${ }^{4,5}$ Clearly, there are complex interactions at the copper surface between additives such as MPSA and chloride.

The objective of this work is a molecular-level understanding of how MPSA inhibits and, in the presence of chloride ion, accelerates copper electrodeposition. This work presents results of molecular dynamics (MD) simulations of MPSA near a copper surface. We examine both atomically flat (111) and rough copper surfaces. We examine how chloride affects the simulated adsorption energy of MPSA and its interaction with copper ions. Furthermore, we compare these simulation results to results from experiments more fully described in a companion study. ${ }^{8}$ In order to assess the role of the chemical endgroups of MPSA, we also included in the simulations (and experiments) two chemical variants of MPSA: 1,3-propanedithiol (PDT) and 1,3-propanedisulfonic acid (PDSA). As discussed in the Conclusion, the simulations suggest that the addition of $\mathrm{NaCl}$ attenuates the adsorption strength of MPSA to the copper surface and the degree of association between MPSA and copper ions. These changes appear to favor an increased deposition rate.

\section{INTERMOLECULAR POTENTIALS}

MD simulations are used in this work to simulate the adsorption behavior of MPSA-like additives at the coppersolution interface, but we note upfront that our model does 
not include deposition reactions; i.e., electron transfer events and incorporation of copper atoms into the surface. At a typical current density of $10 \mathrm{~mA} / \mathrm{cm}^{2}$, a copper deposition "event" occurs on average only every $356 \mu$ s on a nominal $3 \times 3 \mathrm{~nm}^{2}$ surface. This means that on molecular time and length scales the reaction is a rare event and can be neglected if one is instead interested in the necessary molecular adsorption events leading up to the reaction. The continuous competition between solution species for energetically favorable positions near the surface contributes in large measure to the dynamics and structure of the interface. This competition of solution species for favorable sites may be accurately simulated at the nanosecond time scales treated in this and other studies, ${ }^{9-12}$ though proton and electron transfer effects on the simulated interfacial double layer are of increasing interest. ${ }^{13}$ For simplicity our model also does not include proton exchange events through the water solvent. Throughout this work we denote the deprotonated form of MPSA $\left[\mathrm{SO}_{3}-\left(\mathrm{CH}_{2}\right)_{3}-\mathrm{SH}\right]$ with the acronym MPSA ${ }^{-}$and the deprotonated form of PDSA $\left[\mathrm{SO}_{3}-\left(\mathrm{CH}_{2}\right)_{3}-\mathrm{SO}_{3}\right]$ with PDSA $^{2-}$.

Even with these simplifications in the model, substantial effort was required to generate accurate interaction potentials between all the species, including the metal surface. In addition to MPSA-like species, the simulated electrodeposition bath included water and sodium, chloride, bisulfate, cupric, and cuprous ions. Thus a substantial amount of this paper is devoted to a discussion of how these intermolecular potentials were derived. Interactions between two species in solution are treated independently from interactions between solution species and the metal surface. In both cases a simplepoint-charge (SPC) model was used for the solution species. This means that atoms (or groups of atoms) were modeled as sites with a Coulombic point charge and a van der Waalstype interaction center. A united-atom scheme was used where the hydrogen atoms of each methyl group were lumped with the associated carbon.

\section{A. Solution species}

As reported below, suitable potential parameters were found in the literature for water, sodium ion, chloride ion, and cuprous ion. Potential parameters and geometries for the remaining species were obtained from $a b$ initio calculations. Species intramolecular geometry optimizations were performed at the Hartree-Fock level of theory with the 6-31 $+\mathrm{G}(d)$ basis set using the program GaUSSIAN98. ${ }^{14}$ Dihedralangle energy scans were performed at the same calculation level. Species dimer (supermolecular) energies were obtained also using the same basis set, but at the MP2 level of theory. However, copper ions were treated using a split valence RECP basis set, augmented with a single set of $f$-type polarization functions. ${ }^{15,16}$ In each case the dimer energies relative to the energy at infinite separation were calculated at multiple separation distances. The internal geometries of molecules were frozen and not reoptimized during the dimer potential scans. The counterpoise correction to the basis-setsuperposition error was not used. ${ }^{17}$ The correction was, however, calculated for select points in the potential energy scans
TABLE I. Lennard-Jones parameters and charges for each of the monatomic ions in the simulated solution.

\begin{tabular}{lccc}
\hline \hline & $\sigma, \AA$ & $\epsilon / k, \mathrm{~K}$ & $q,|e|$ \\
\hline $\mathrm{Na}^{+}$ & 2.35 & 55.30 & +1 \\
$\mathrm{Cl}^{-}$ & 4.42 & 54.20 & -1 \\
$\mathrm{Cu}^{+}$ & 1.55 & 50.32 & +1 \\
$\mathrm{Cu}^{2+}$ & 1.55 & 50.32 & +2 \\
\hline \hline
\end{tabular}

and was found to be negligible except in one case discussed below. Coulombic site charges for solution species were assigned to reproduce the electrostatic field about each molecule, including the dipole moment, using the CHelpG scheme. The electrostatic field was calculated using a larger $6-311+\mathrm{G}(2 d f, 2 p d)$ basis set with MP2 level of theory.

For interactions between solution species we used the Lennard-Jones (LJ) potential to represent van der Waals interactions. The SPC dimer energy, including LJ and Coulombic terms, was fitted to the ab initio dimer energy by varying $\sigma$ and $\epsilon$ values not available from prior work. In all cases the Wheeler-Rowley combining rules were used to determine cross interactions between unlike sites. ${ }^{18}$

\section{Water and ions}

The simple-point-charge/extended ${ }^{19}$ (SPC/E) potential model was used for water. It reproduces fairly accurately the properties of water at ambient temperature and pressures. ${ }^{19}$ Table I shows the parameters used for each of the monatomic ions in solution. Note that the well depths are very similar, but there are significant differences in the size parameter $\sigma$. This size parameter and the charge greatly influence the ion hydration. Generally speaking, the smaller the size, the greater the strength of hydration.

Sodium and chloride potential parameters were obtained from Wheeler and Newman. ${ }^{20}$ The parameters have been shown to be moderately successful at matching a range of equilibrium and transport properties for aqueous solutions.

In contrast to sodium and chloride, there are relatively few models of aqueous copper ions reported in the literature. Fulton et al. reported LJ parameters for cuprous ion used in simulating $\left[\mathrm{CuBr}_{2}\right]^{-}{ }^{21}$ For simplicity and consistency we modeled both $\mathrm{Cu}(\mathrm{I})$ and $\mathrm{Cu}(\mathrm{II})$ using the Fulton $\mathrm{LJ}$ parameters, with the ions differing only in Coulombic charge.

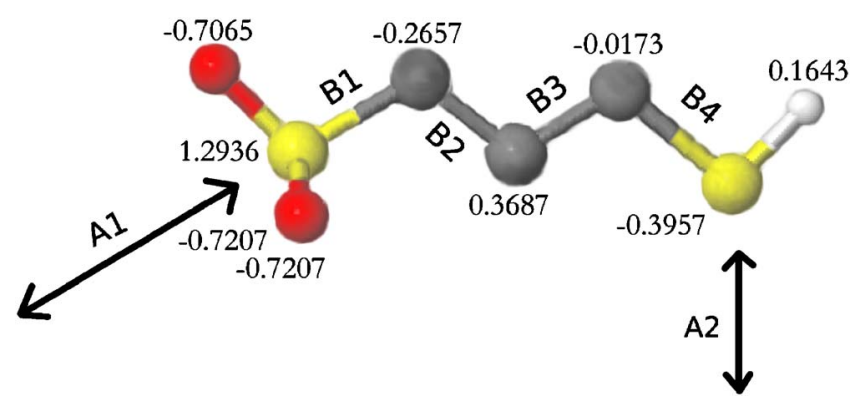

FIG. 1. (Color online) Pictorial representation of MPSA ${ }^{-}$. A1 and A2 represent the lines of approach used to regress interaction parameters with A1 approaching the sulfonate group and A2 the sulfide group. B1-B4 label the bonds about which dihedral potentials have been calculated. Site charges, in units of $|e|$, are also indicated. 


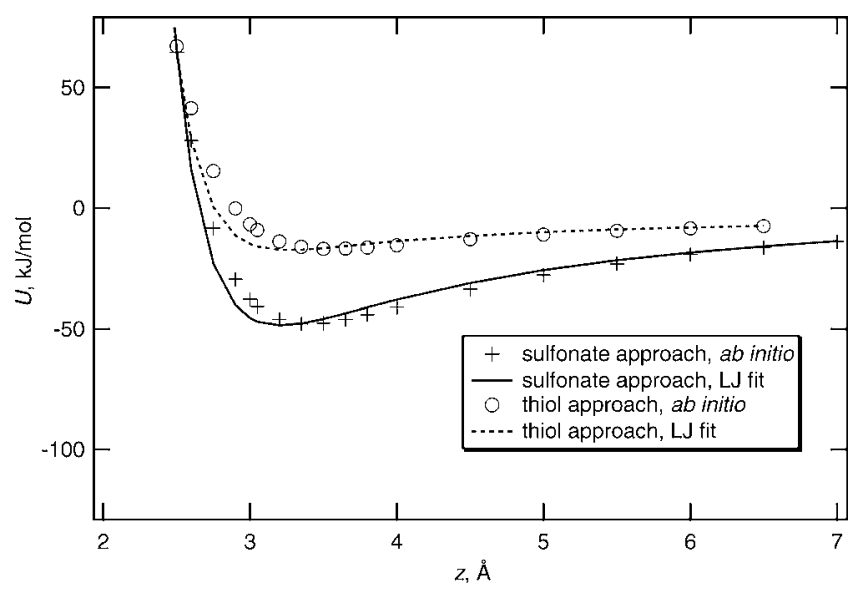

FIG. 2. Pairwise interaction of MPSA with water. Distances are measured from the aqueous oxygen atom to the respective sulfur atom, with approach vectors indicated in Fig. 1. The water dipole moment points toward MPSA along the same vectors.

\section{MPSA-}

MPSA $^{-}$was modeled with nine interacting sites shown in Fig. 1: four sites constitute the sulfonate group, three methyl-type sites constitute the carbon backbone, and two sites constitute the thiol group. Also shown in the figure are the accompanying Coulombic site charges and additional labels.

It is important to realize that when two charged species approach each other, the potential is Coulombically dominated. That is, the magnitude of the effective LJ dispersion $\left(r^{-6}\right)$ term is small relative to the charge-charge interaction. For example, the Coulombic interaction between a sodium ion and a sulfonate oxygen is 50 times larger than the dispersive interaction at $2.5 \AA$. In such cases, the potential effectively consists of a charge-charge interaction and a Pauli repulsion term. In practice, this means that one of the LJ parameters can be fixed and not used in the fit if desired. For each of the sites on the organic molecules for which we regress LJ interaction parameters, we fix the $\epsilon$ parameter to values found in the literature. The $\epsilon$ values for sulfur and oxygen (in the thiol, bisulfate, or sulfonate groups) are given by Vishnyakov and Neimark, ${ }^{22}$ and the $\epsilon$ parameter for thiol

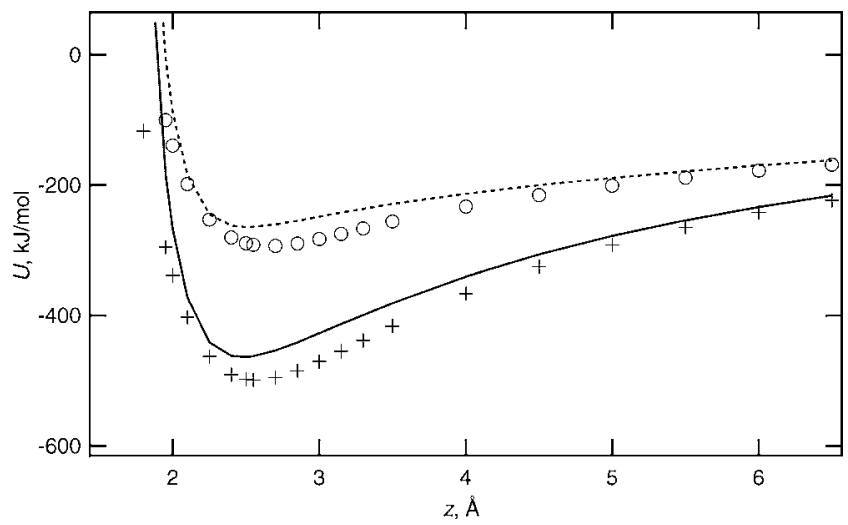

FIG. 3. Pairwise interaction of $\mathrm{MPSA}^{-}$with $\mathrm{Na}^{+}$. Distances are measured from the $\mathrm{Na}^{+}$to the respective sulfur atom, with approach vectors indicated in Fig. 1. Symbols and lines have the same meanings as in Fig. 2.

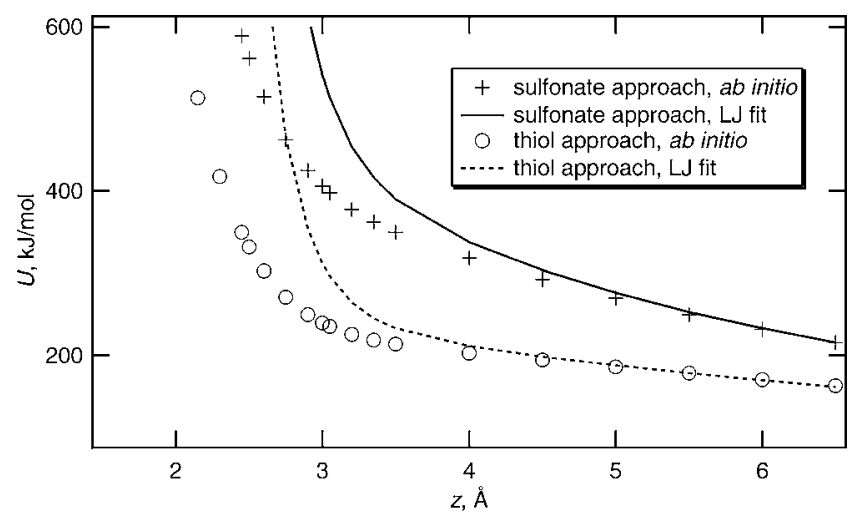

FIG. 4. Ab initio potential scans of $\mathrm{MPSA}^{-}$with $\mathrm{Cl}^{-}$. Distances are measured from the $\mathrm{Cl}^{-}$to the respective sulfur atom, with approach vectors indicated in Fig. 1.

sulfur was left at the OPLS value. ${ }^{23}$ In addition, OPLS parameters of Jorgensen and co-workers were used for the $\mathrm{CH}_{2}$ sites. $^{23,24}$

The reason for not using literature $\mathrm{LJ}$ values entirely was the desire to improve the quality of the critical interaction of the thiol and sulfonate end groups of $\mathrm{MPSA}^{-}$with other species. We therefore regressed LJ parameters for these sites on MPSA ${ }^{-}$by calculating interactions of $\mathrm{MPSA}^{-}$with water, sodium, chloride, and cuprous species for multiple positions along the $\mathrm{A} 1$ and $\mathrm{A} 2$ lines of approach in Fig. 1. Like the hydrogen sites on SPC/E water, $\epsilon=0$ for the sulfide hydrogen site. This is consistent with the modeling of Kaminski et al. of the thiol group in cysteine. ${ }^{23}$

The results from the $a b$ initio potential scans are shown in Figs. 2-5. The points are the quantum-mechanical results and the curves are the model fits (representing the cumulative effect of site-site Coulombic and LJ interactions). It is important to note that we are not regressing additional sodium, chloride, water, or cuprous ion LJ parameters for interactions with MPSA ${ }^{-}$. Using the combining rules we globally fit LJ parameters for MPSA sites only. The LJ fit of the $a b$ initio data matches very well in the case of water, shown in Fig. 2. This is encouraging as the majority of interatomic interactions involving MPSA $^{-}$will be with the solvent, wa-

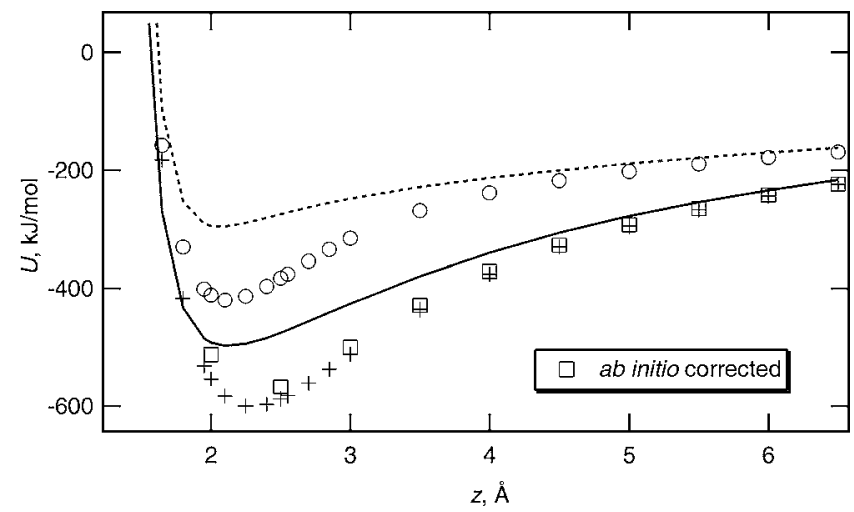

FIG. 5. Ab initio potential scans of MPSA with $\mathrm{Cu}^{+}$. Distances are measured from the $\mathrm{Cu}^{+}$to the respective sulfur atom, with approach vectors indicated in Fig. 1. The $a b$ initio corrected points reflect the small basis-setsuperposition error. Symbols and lines retain the same meanings as in Fig. 2 or 4. 
TABLE II. Dihedral potential parameters for the sulfur-containing molecules, in units of K. B1-B4 correspond to the bonds indicated in Fig. 1 and B5 corresponds to the single dihedral angle in bisulfate.

\begin{tabular}{lccccc}
\hline \hline & B1 & B2 & B3 & B4 & B5 \\
\hline$A_{0}$ & 1181 & 482.5 & 1601 & 567.6 & 288.5 \\
$A_{1}$ & -3596 & -802.6 & -2150 & -1174 & -398.4 \\
$A_{2}$ & -12.14 & 4763 & 917.1 & -415.0 & -266.1 \\
$A_{3}$ & 4795 & 5000 & 5030 & 1333 & 423.9 \\
$A_{4}$ & 13.72 & -1059 & 785.3 & 61.19 & -3.045 \\
$A_{5}$ & -0.87 & 47.13 & 435.9 & -58.08 & -5.158 \\
\hline \hline
\end{tabular}

ter. When water approaches the sulfonate head group, the most favorable interaction is about $40 \mathrm{~kJ} / \mathrm{mol}$ more negative than when approaching the thiol group.

Figures. 3-5 show potential energy curves as a function of distance when the respective ions approach each end of the MPSA ${ }^{-}$molecule. The globally fit SPC models and the $a b$ initio results agree within $5 \%$ at distances greater than about $4 \AA$ At At shorter distances MPSA ${ }^{-}$is able to polarize and thus significantly enhance the attraction $\left(\right.$ for $\mathrm{Cu}^{+}$) or decrease the repulsion (for $\mathrm{Cl}^{-}$). This polarization effect is only partially accounted for in the pairwise Lennard-Jones potential. Figure 5 contains additional points to illustrate our general observation that the counterpoise correction is small relative to the total interaction.

The combining rules and the regressed MPSA ${ }^{-}$parameters do not fit the well depth of the cuprous-MPSA ${ }^{-}$scans particularly well. However, it is possible that the ab initio result overpredicts the interaction of $\mathrm{Cu}^{+}$with $\mathrm{MPSA}^{-}$. The unexpectedly large observed difference between the $\mathrm{Na}^{+}$and $\mathrm{Cu}^{+}$ab initio energies at the respective well minima is cause for concern. Moreover, cupric ion-MPSA ${ }^{-}$scans are not included in the regression of the LJ solution parameters for MPSA $^{-}$as we had difficulty obtaining realistic ab initio results for the $\mathrm{Cu}^{2+}$-MPSA scans. The $\mathrm{Cu}^{+}-\mathrm{Cu}_{10}$ cluster scans presented in the section below also show somewhat unexpected results. Our results suggest that better quantummechanical models describing the interactions of cupric and cuprous ions with other species are needed.

The bonds about which the MPSA ${ }^{-}$dihedral angles can change are indicated in Fig. 1. The form of the fitted dihedral potential used in the MD simulations is

$$
U_{\mathrm{dih}}=\sum_{i=0}^{5} A_{i}[\cos (\theta)]^{i}
$$

where $\theta$ is the dihedral angle and the $A_{i}$ are potential parameters. The fitted energy does not vary by more than $3 \mathrm{~kJ} / \mathrm{mol}$ from the quantum-chemical results. Table II contains the dihedral potential parameters $A_{i}$ for the relevant species. The same set of parameters is used for the MPSA${ }^{-}$, PDT, and PDSA $^{2-}$ molecules.

\section{PDT and PDSA ${ }^{2-}$}

As shown in Fig. 1, MPSA ${ }^{-}$has a sulfonate group and a thiol group at the ends of a propane chain. In contrast the two groups flanking the propane chain are both thiol for PDT and both sulfonate for $\mathrm{PDSA}^{2-}$. Experimentally, we have investigated how these molecular changes affect the observed in- hibition or acceleration of copper deposition. Molecular simulations analogous to the substitution experiments were conducted as part of this work. ${ }^{8}$

We used the MPSA ${ }^{-}$-LJ parameters between the corresponding sites in PDT and PDSA ${ }^{2-}$. However, the Coulombic charges are unique to each molecule. The site charges for PDT and PDSA ${ }^{2-}$ were found in the same manner as MPSA ${ }^{-}$ as previously described. Listed in Table III are the charge and model coordinates for all the sulfur-containing molecules. The charges on PDT are symmetric due to the $C_{2 V}$ symmetry of PDT. PDSA ${ }^{2-}$ belongs to the $C_{2}$ point group and the site charges on opposite halves of the molecule differ by less than $3 \%$.

\section{Bisulfate}

No new parameter values were regressed for the bisulfate model. Table IV summarizes the LJ parameters for the sulfur-containing species in solution.

Table III gives the coordinates and charges for the bisulfate ion model. The dihedral angle defined by the oxygensulfur bond is modeled with the potential given in Eq. (1) and the constants given in Table II.

As a check on the use of previously obtained parameters for bisulfate, we calculated dimer interactions between two bisulfate ions and between a bisulfate and a water. The water routes are illustrated in Fig. 6 and the results are given in Fig. 7. Reasonable agreement between $a b$ initio and our model potentials were obtained. In particular, for the attractive routes (routes 1 and 4) the SPC model agrees well with the $a b$ initio results.

\section{B. Copper surface-interaction potentials}

Potential parameters between species in solution (bisulphate, sulfate, MPSA${ }^{-}$, and $\mathrm{Cu}^{+}$) and the copper metal surface were obtained from $a b$ initio calculations with the surface modeled as a ten-atom copper cluster. We previously reported similar results for water, $\mathrm{Na}^{+}$, and $\mathrm{Cl}^{-}$interacting with a copper cluster. ${ }^{25} \mathrm{MP} 2 / 6-31+\mathrm{G}(d)$ calculations were used for all species except copper atoms and ions, which as before used a split valence RECP basis set augmented with a single set of $f$-type polarization functions. ${ }^{15,16}$

The SPC model used for copper-solution species interactions has two main distinctions. First, the van der Waalstype interactions were fitted to modified-Morse potentials applied on a site-pairwise basis, consistent with earlier work. That is, each copper metal atom was treated as an interaction center. The modified-Morse potential basically makes up the 
TABLE III. Site charges and initial coordinates for the sulfur-containing molecules. Dihedral angles are allowed to vary during the simulation; all other bond distances and angles are fixed.

\begin{tabular}{|c|c|c|c|c|}
\hline & $q,|e|$ & $x, \AA$ & $y, \AA$ & $z, \AA$ \\
\hline \multicolumn{5}{|l|}{ MPSA $^{-}$} \\
\hline $\mathrm{H}$ & 0.1643 & 0.0000 & -0.8823 & 0.0000 \\
\hline S & -0.3957 & 0.0000 & 0.1216 & 0.8794 \\
\hline $\mathrm{CH}_{2}$ & -0.0173 & 0.0000 & -0.9016 & 2.3931 \\
\hline $\mathrm{CH}_{2}$ & 0.3687 & 0.0000 & 0.0000 & 3.6150 \\
\hline $\mathrm{CH}_{2}$ & -0.2657 & 0.0000 & -0.8219 & 4.8919 \\
\hline $\mathrm{S}$ & 1.2936 & 0.0000 & 0.2246 & 6.3637 \\
\hline $\mathrm{O}$ & -0.7207 & 1.2321 & 1.0126 & 6.2377 \\
\hline $\mathrm{O}$ & -0.7207 & -1.2321 & 1.0126 & 6.2377 \\
\hline $\mathrm{O}$ & -0.7065 & 0.0000 & -0.7292 & 7.4770 \\
\hline \multicolumn{5}{|l|}{ PDT } \\
\hline $\mathrm{H}$ & 0.2049 & 0.0000 & -0.5771 & 0.0000 \\
\hline S & -0.3309 & 0.0000 & 0.3879 & 0.9204 \\
\hline $\mathrm{CH}_{2}$ & -0.1159 & 0.0000 & -0.7050 & 2.3766 \\
\hline $\mathrm{CH}_{2}$ & 0.4838 & 0.0000 & 0.1581 & 3.6282 \\
\hline $\mathrm{CH}_{2}$ & -0.1159 & 0.0000 & -0.7050 & 4.8797 \\
\hline $\mathrm{S}$ & -0.3309 & 0.0000 & 0.3878 & 6.3359 \\
\hline $\mathrm{H}$ & 0.2049 & 0.0000 & -0.5772 & 7.2563 \\
\hline \multicolumn{5}{|l|}{$\mathrm{PDSA}^{2-}$} \\
\hline $\mathrm{O}$ & -0.7797 & 0.0000 & -1.1428 & 0.0000 \\
\hline $\mathrm{O}$ & -0.7560 & 1.2054 & 0.6797 & 1.0009 \\
\hline $\mathrm{O}$ & -0.7560 & -1.2054 & 0.6797 & 1.0009 \\
\hline S & 1.3791 & 0.0000 & -0.1081 & 1.0015 \\
\hline $\mathrm{CH}_{2}$ & -0.2277 & 0.0000 & -1.0193 & 2.5539 \\
\hline $\mathrm{CH}_{2}$ & 0.2709 & 0.0000 & -0.1945 & 3.8414 \\
\hline $\mathrm{CH}_{2}$ & -0.2141 & 0.0000 & -1.1279 & 5.0524 \\
\hline $\mathrm{S}$ & 1.4175 & 0.0000 & -0.3703 & 6.6852 \\
\hline $\mathrm{O}$ & -0.7640 & 0.0000 & 1.0566 & 6.4911 \\
\hline $\mathrm{O}$ & -0.7850 & -1.1990 & -0.8586 & 7.3156 \\
\hline $\mathrm{O}$ & -0.7850 & 1.1990 & -0.8586 & 7.3156 \\
\hline \multicolumn{5}{|l|}{$\mathrm{HSO}_{4}^{-}$} \\
\hline S & 1.4285 & -0.0720 & -0.0720 & 0.7769 \\
\hline $\mathrm{O}$ & -0.6958 & -0.8721 & 0.8423 & 0.0001 \\
\hline $\mathrm{O}$ & -0.6958 & 0.8424 & -0.8720 & 0.0000 \\
\hline $\mathrm{O}$ & -0.7472 & -0.8041 & -0.8042 & 1.7968 \\
\hline $\mathrm{O}$ & -0.6651 & 0.8913 & 0.8914 & 1.6808 \\
\hline $\mathrm{H}$ & 0.3754 & 0.6916 & 0.6917 & 2.5863 \\
\hline
\end{tabular}

difference between the $a b$ initio energy and the strictly Coulombic interactions. Second, the Coulombic charges on the copper atoms are allowed to fluctuate or move fluidly from one metal atom to another, in response to the local electrostatic environment and subject to a total-charge constraint. This scheme, termed electrode charge dynamics (ECD), al-

TABLE IV. Solution-phase LJ parameters for sulfur-containing species.

\begin{tabular}{llc}
\hline \hline & $\sigma, \AA$ & $\epsilon / k, \mathrm{~K}$ \\
\hline $\mathrm{S}$ & 3.405 & 125.9 \\
$\mathrm{~S}_{\text {thiol }}$ & 3.407 & 213.9 \\
$\mathrm{O}_{\text {sulfonate }}$ & 3.054 & 100.7 \\
$\mathrm{O}_{\text {sulfate }}$ & 2.537 & 100.7 \\
$\mathrm{CH}_{2}$ & 3.905 & 59.38 \\
$\mathrm{H}$ & 0 & 0 \\
\hline \hline
\end{tabular}

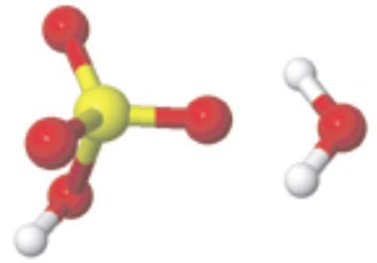

Route 1
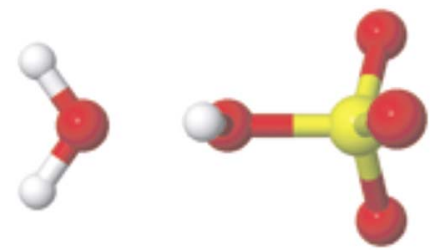

Route 3

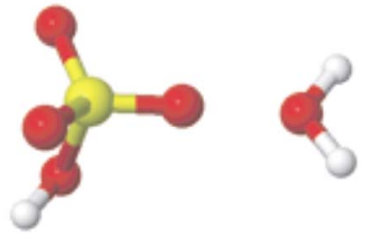

Route 2

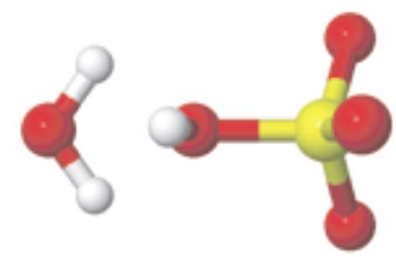

Route 4
FIG. 6. (Color online) Depictions of the two attractive and two repulsive routes. In each route, one sulfur and two oxygen atoms are colinear.

lows the metal surface to polarize as described in our prior work. ${ }^{25}$ ECD is used both in the fitting scheme (with a tenatom cluster) and for the large-scale MD simulations and allows the small-cluster calculations to be realistically extrapolated to the larger size.

The modified-Morse parameters for the surface interactions of $\mathrm{Cl}^{-}, \mathrm{Na}^{+}$, and water were reported previously. ${ }^{25}$ The parameters for aqueous hydrogen atoms were also used in this work to describe the interactions between sulfur-based hydrogen atoms and the copper surface.

Two routes of approach for $\mathrm{Cu}_{10}$-bisulfate were calculated and are labeled and shown pictorially in Fig. 8. The interaction potential is shown in Fig. 9. In each case the sulfur atom is directly over the top site of the cluster. The $a b$ initio data are well represented by the ECD fit.

It was too costly to simulate the full $\mathrm{MPSA}^{-}$molecule approaching the copper cluster. Therefore, we used two proxies for the endgroups, a methyl thiol $\left(\mathrm{HS}-\mathrm{CH}_{3}\right)$ and a sulfonate ion $\left(\mathrm{HSO}_{3}^{-}\right)$, to determine the surface potentials. We were able to include a few points with the whole MPSA $^{-}$ approaching the surface for comparison. Pictorial representations of the thiol, sulfonate, and MPSA ${ }^{-}$approaches are shown in Fig. 10. Not shown is the MPSA ${ }^{-}$approach when the thiol group is closest to the surface. Such an approach is

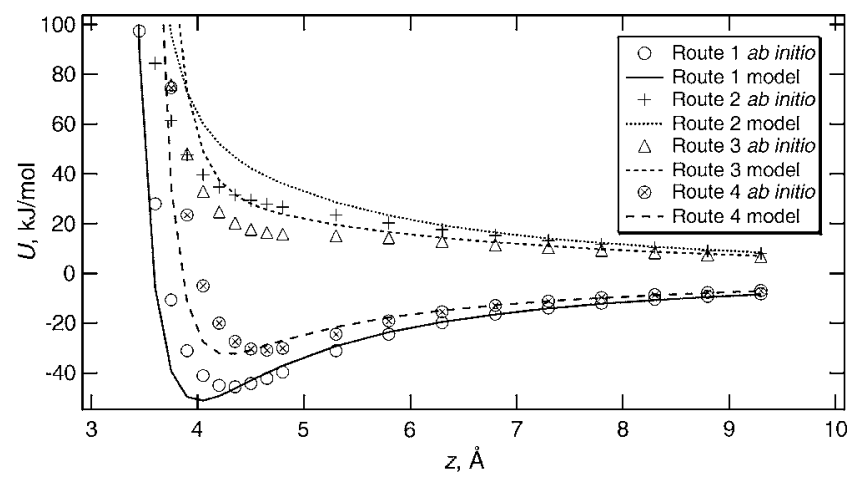

FIG. 7. Ab initio and model potential energy of water-bisulfate scans indicated in Fig. 6. Distance $z$ is the principal sulfur-oxygen separation. 


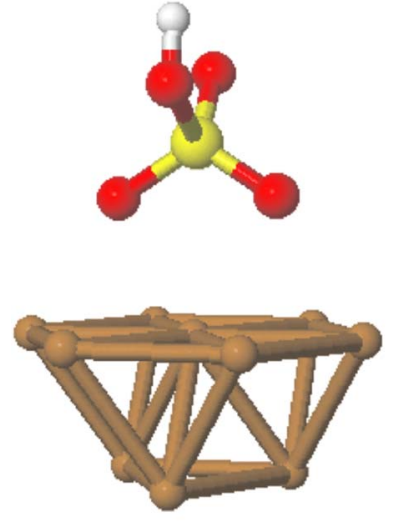

Route 1

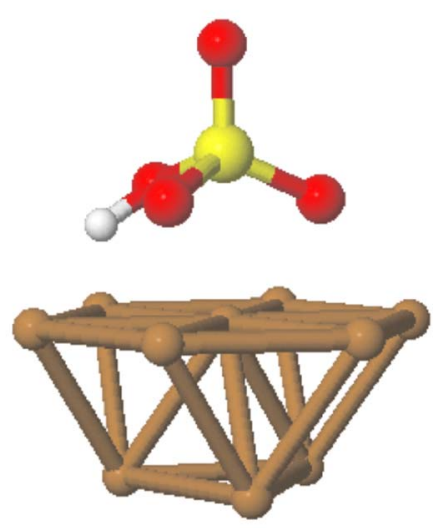

Route 2
FIG. 8. (Color online) Pictorial representation of bisulfate ion approaching a $\mathrm{Cu}_{10}$ cluster.

equivalent to the methyl thiol approach but with the rest of the MPSA ${ }^{-}$included. In each approach a sulfur atom is directly above the top site.

In our fitting procedure we found that a transferable set of modified-Morse parameters for sulfur and oxygen atoms worked well. Figure 11 shows that our potential model reproduces the $a b$ initio result. The sulfonate approach is more energetically favored than the thiol approach as the highly charged sulfonate group is closer to the surface. Including the entire $\mathrm{MPSA}^{-}$molecule appears to slightly decrease the $a b$ initio interaction energy.

An approximation of the cuprous ion potential approaching a $\mathrm{Cu}_{10}$ cluster was obtained from preliminary $a b$ initio calculations. ${ }^{26}$ In this particular case the calculated energies did not properly converge toward zero at large separation distances but appear more accurate at the contact point of the ion with the surface. Further work is required to generate a more robust calculation of the potential energy of $\mathrm{Cu}^{+}$and $\mathrm{Cu}^{2+}$ species interacting with a copper surface. For the present work the modified-Morse parameters for these two ions were taken to be the same and are given in Table V.

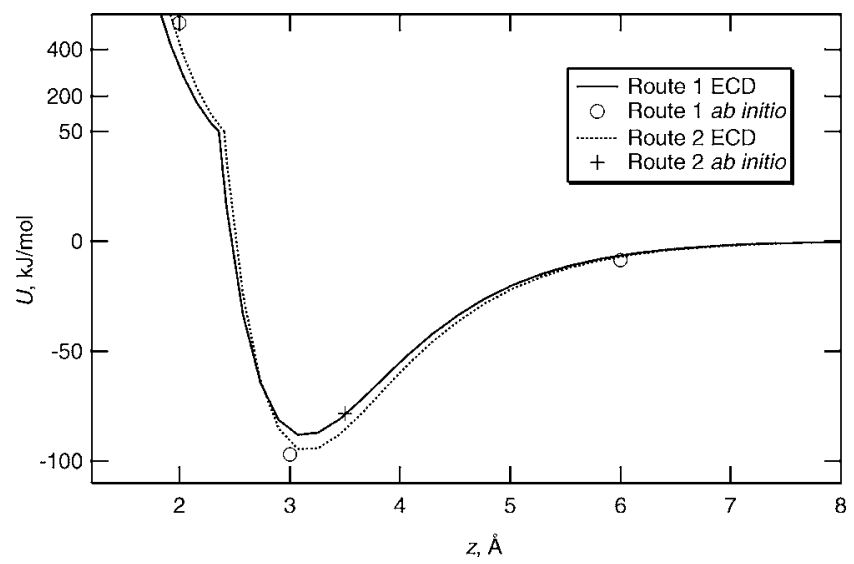

FIG. 9. Potential energy of bisulfate ion approaching a $\mathrm{Cu}_{10}$ cluster. Distances are measured from the sulfur atom to the uppermost copper layer. The apparent change in slope at $50 \mathrm{~kJ} / \mathrm{mol}$ is due to a scale change in the ordinate.

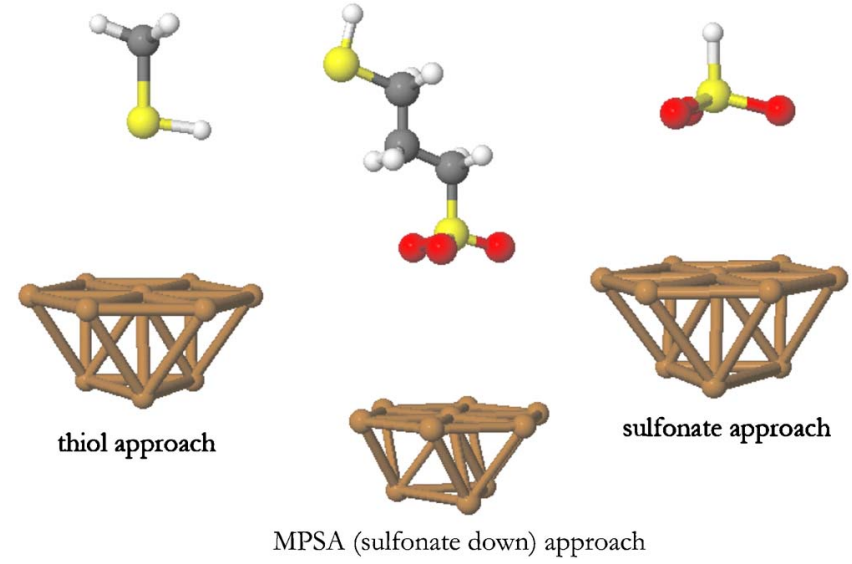

FIG. 10. (Color online) Pictorial representations of MPSA ${ }^{-}$and derivatives approaching a $\mathrm{Cu}_{10}$ cluster. In each approach a sulfur atom is directly above the central top site.

\section{Accuracy of the fitted potentials for liquid simulations}

The $a b$ initio calculations reported above are for interactions in vacuum rather than in a condensed phase where multibody effects could be important. The interactions involving water should be reasonably accurate because the dipole moment of SPC/E water is adjusted to represent condensed-phase polarization. The fixed charges assigned to the sulfur-containing species have not been adjusted to the condensed phase. Multibody Coulombic effects are explicitly included in the copper-surface model, allowing the copper atoms to respond realistically to a condensed-phase environment. Thus the molecular models have some deficiencies but should largely capture the intended condensed-phase behavior.

We also note that the quantum-mechanical calculations reported here do not include corrections for basis-set superposition error (BSSE). The magnitude of the error depends strongly on the electron structure of the interacting species. As of yet, there is not a viable scheme to properly correct the error in strongly interacting systems. ${ }^{27}$ One commonly used method to estimate BSSE is the counterpoise correction scheme, which has been used in some studies of metal

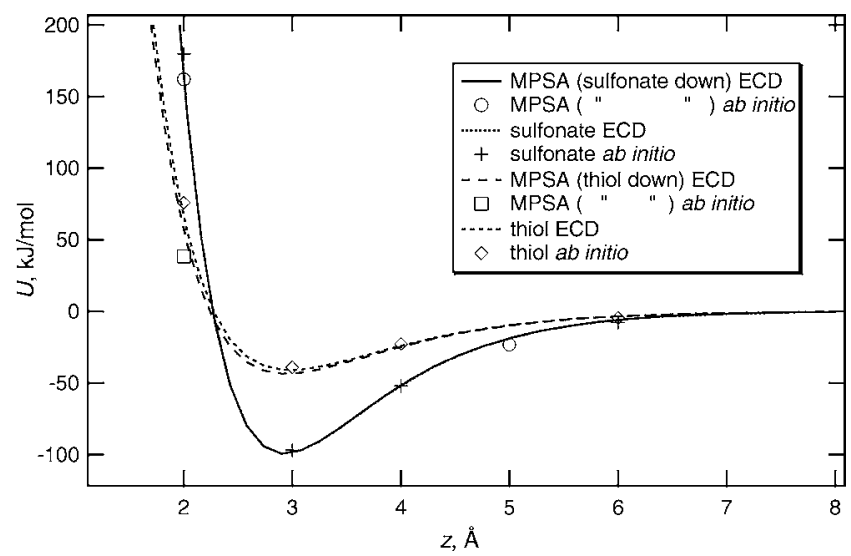

FIG. 11. Potential energy of MPSA ${ }^{-}$and derivatives approaching a $\mathrm{Cu}_{10}$ cluster. Distance $z$ is measured from the primary sulfur site to the closest copper atom. 
TABLE V. Modified-Morse Cu-metal interaction parameters. Parameters for $\mathrm{Cl}^{-}, \mathrm{Na}^{+}, \mathrm{H}$, and $\mathrm{O}$ sites are given in Ref. 25 .

\begin{tabular}{lccc}
\hline \hline & $\epsilon, \mathrm{kJ} / \mathrm{mol}$ & $A, \AA^{-1}$ & $r^{*}, \AA$ \\
\hline $\mathrm{Cu}$ ions & 40.0 & 1.0 & 3.1 \\
$\mathrm{~S}$ & 6.0 & 1.1 & 3.5 \\
$\mathrm{O}_{s}{ }^{\mathrm{a}}$ & 3.1 & 1.35 & 3.2 \\
$\mathrm{CH}_{x}$ & 2.0 & 1.3 & 3.2 \\
\hline \hline
\end{tabular}

axygen atoms bonded to a sulfur atom.

clusters $^{28,29}$ and not in others. ${ }^{30-32}$ Ruuska et al. suggested this scheme may overestimate the BSSE for metal clusters. ${ }^{16}$

\section{MD SIMULATION DETAILS}

Molecular dynamics simulations were used to obtain effective adsorption/desorption profiles of several species approaching the copper surface, either flat (111) or saw toothed, with and without sodium and chloride ions present. In the simulations reported here, 1000 molecules are simulated in a unit cell of approximately $80 \times 20 \times 22 \AA^{3}$ in the $x$, $y$, and $z$ directions, respectively, with 10 (flat) or 11 (sawtooth surface) layers of copper atoms present. Shown in Fig. 12 is a representation of the unit cell with a sawtoothed surface.

When simulating an organic additive (MPSA, PDT, or PDSA) near a copper (111) surface, we simulate one molecule of the additive in a water bath of 1000 total molecules. There are 36 bisulfate ions, 12 cupric ions, and 12 cuprous ions, and when $\mathrm{NaCl}$ is present there are $24 \mathrm{Na}^{+}$and $24 \mathrm{Cl}^{-}$ ions in the solution. The simulations occur within the canonical ensemble at a temperature of $298.15 \mathrm{~K}$. Each simulation of a forced adsorption and desorption event lasts $3.7 \mathrm{~ns}$ (timesteps of size $2 \mathrm{fs}$ ). Each simulation was equilibrated for approximately $100 \mathrm{ps}$ prior to collection of the adsorption and desorption data. This included an initial period over which the volume of the cell is adjusted so that the density of water in the center third of the fluid region was approximately $55 \mathrm{~mol} / 1$. The reported adsorption/desorption profiles are averages over 10-12 unique simulations.

Coulombic interactions are treated using the particleparticle-particle-mesh Ewald methodology. ${ }^{33-35}$ Short-range interactions are cut off at $10 \AA$. The charges of the copper metal atoms dynamically adjust to the adjacent liquid structure according to the electrode-charge-dynamics (ECD) routine. $^{25}$

An electrostatic potential difference of $3.6 \mathrm{~V}$ is maintained between the two bounding copper surfaces in order to

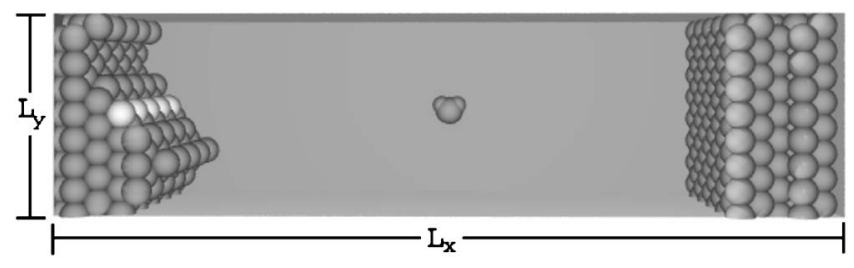

FIG. 12. 3D representation of simulation cell with sawtooth surface. A single water molecule, shown to indicate scale, is located in the middle of the fluid region. The lightly shaded surface copper atoms indicate the row at which adsorption/desorption events are recorded. generate an electric potential on the negative electrode that is close to experimental electrodeposition conditions. Adsorption and desorption of additives took place on the negative electrode.

We obtained the adsorption energies of $\mathrm{H}_{2} \mathrm{O}$ and $\mathrm{MPSA}^{-}$ by forcing the molecule toward and then away from the surface. The species begins its forced movement $8 \AA$ from the surface. At discrete locations along its route, the species is held at a given position by artificially imposing a harmonic potential constraint on its location. How the species responds to the constraint is recorded and used to obtain the energy of adsorption or desorption. In this paper, we use the terms adsorption or binding energy to signify the energy difference between the separated and proximal states of a molecular species to a surface or to a different molecular group, with a negative energy signifying a favorable proximal orientation relative to the separated state.

The overall ionic concentrations $(\approx 0.5 M)$ in the simulated solution are in excess of typical experimental conditions (millimolar) due to dilution constraints when treating solvent molecules explicitly. Concentration effects are not expected to impact the qualitative effects of $\mathrm{NaCl}$ addition on $\mathrm{MPSA}^{-}$adsorption being studied in our simulations. Also, we chose to simulate an equal number of cupric and cuprous ions as an approximation to experimental interfacial concentrations of $\mathrm{Cu}^{+}$and $\mathrm{Cu}^{2+}$. Depending on the rate of copper deposition or dissolution, the actual experimental interfacial ratio of the number of cupric to cuprous ions will vary.

\section{SIMULATION RESULTS}

We report the following simulation results: (1) the ion density profiles near the flat copper surface, (2) the energetics of organic species adsorption and desorption, (3) the distribution of cupric and cuprous ions around MPSA${ }^{-}$, and (4) the valence-charge work function of the metal surfaces. Quantitative values as well as statistical significances in the difference between values are reported at a $95 \%$ confidence interval, unless stated otherwise. The Conclusions section discusses how the simulated observations provide insight into possible mechanisms explaining the inhibition and acceleration of copper electrodeposition in the presence of MPSA.

Experimentally it is known that thiol-containing additives such as MPSA can inhibit copper deposition. ${ }^{36}$ This may occur as the additive binds to the active surface sites and thus blocks cation transfer to the metal surface. Subsequent addition of sodium chloride drastically increases the deposition rate likely due to the changes to the interfacial additive behavior. ${ }^{8,37}$ Consistent with this picture of the surface chemistry, our simulations show that $\mathrm{NaCl}$ reduces the adsorption strength of MPSA and chemically related species. We also find that the addition of sodium chloride reduces the work required to remove surface electrons. This result implies a reduction in the activation energy for copper-ion reduction, also increasing the deposition rate. 


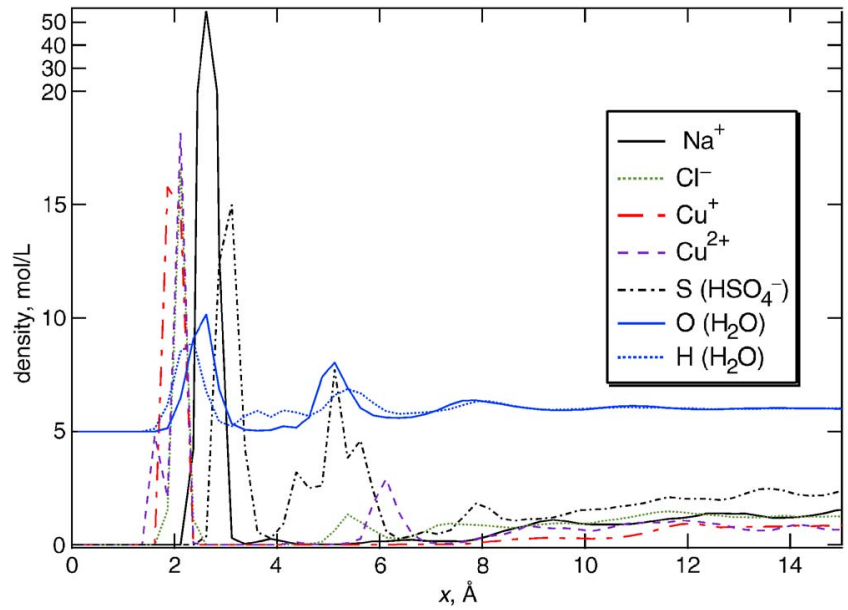

FIG. 13. (Color online) Density of water sites and ions near a negative $\mathrm{Cu}(111)$ surface. The curves corresponding to water density have been normalized and offset for clarity. At small distances the chloride curve is partially obscured by its close overlap with the cupric curve.

\section{A. Water profiles and potential of mean force}

Adsorption and desorption of ions or molecules at the copper surface are strongly dependent on density profiles in the electrochemical double layer. We therefore profile the density of each species as a function of its distance from the wall to understand better the local properties in the interfacial layer. Figure 13 gives ion and solvent densities near the flat (111) negatively charged copper wall without $\mathrm{MPSA}^{-}$ present. The profiles are equilibrium averages over about 16 ns of simulation time. During simulations the net surface charges on the opposing flat (111) electrodes were, respectively, $-0.39 \pm 0.03$ and $0.53 \pm 0.03 \mu \mathrm{C} / \mathrm{cm}^{2}$. In the figure the surface copper atoms are at $x=0 \AA$; the opposing electrode surface begins at $x=58.6 \AA$. Also shown in the figure is the water density profile, which has been normalized by the bulk water density and offset vertically to make the graph more readable.

The structure of water-the most abundant surface species-greatly influences the adsorption and orientational behavior of the ions and additives. In Fig. 13, it is apparent that the orientation of the water layer closest to the surface is with the hydrogen atoms parallel to the surface with a slight tipping of the hydrogen atoms toward the negatively charged surface. A region of low density separates two water layers, with results suggesting that water is primarily bound to other water molecules in the same layer while interactions with adjacent layers play a secondary role. Such two-dimensional water "sheets" near the solid surface have been previously discussed. ${ }^{25,38,39}$

One might expect a significantly higher density of the more highly charged cupric ion at the electrode surface, but surprisingly the $\mathrm{Cu}^{+}$and $\mathrm{Cu}^{2+}$ concentrations are nearly equal in the immediate vicinity of the surface. Stronger hydration of $\mathrm{Cu}^{2+}$ evidently compensates for the larger charge. Inclusion of explicit solvent and other atomistic details is important in obtaining this result. Moreover, the surfacedensity peaks for chloride and copper ions coincide and are of similar magnitude indicating the presence of surface

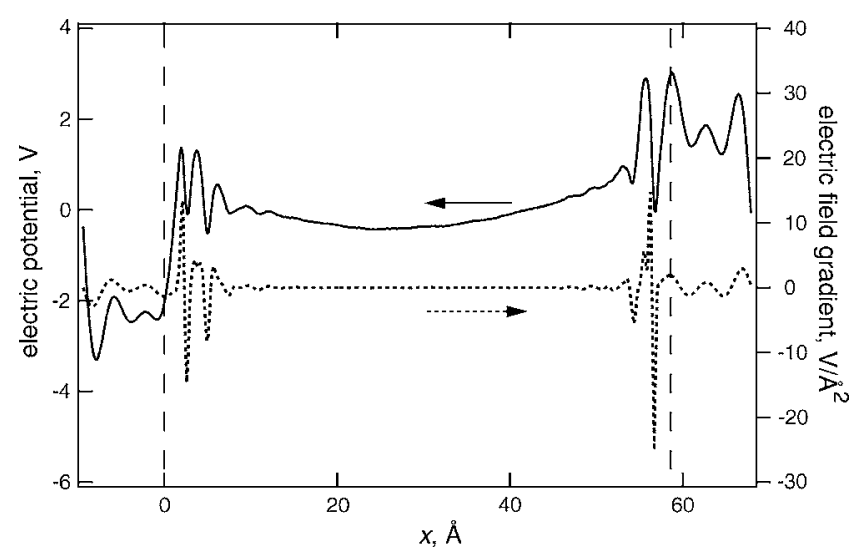

FIG. 14. Electric potential profile and electric-field-gradient profile of the system with charged copper surfaces. The gradient of the electric field is proportional to the charge density in the fluid. The vertical dashed lines indicate the locations of the two surface layers of copper atoms, such that the liquid region is between the two lines.

copper-chloride ion pairs. Copper-chloride complexes are known to inhibit copper electrodeposition and readily precipitate out of solution. 4,40

From the site densities and electrode charge, the electrostatic potential through the fluid can be found using Poisson's equation. The two boundary conditions needed to solve the second order differential equation are the potential at a given point and the average potential difference between the two electrodes. In Fig. 14, the electrostatic potential and the gradient of the electric field are shown. The gradient of the electric field is proportional to the charge density in the fluid. The region between $x=-9.4 \AA$ and $x=0 \AA$ is occupied by the negative electrode; the positive electrode is located between $x=58.6 \AA$ and $x=68.0 \AA$. The fluid region is between $x$ $=0 \AA$ and $x=58.6 \AA$.

From the solution-charge density that is proportional to the electric-field gradient shown in Fig. 14, one can see that a positively charged fluid layer is nearest the negative surface (near $x=0 \AA$ ) and a negatively charged fluid layer is nearest the positive surface (near $x=58.6 \AA$ ). We note the oscillating charged layers in solution near the two surfaces are asymmetric. This outcome correlates with the water density fluctuations near the two unique surfaces, i.e., there are more fluctuations in the water density at the negatively charged interface, as $\mathrm{we}^{41}$ and others have previously observed. $^{42}$

There is an average $3.6 \mathrm{~V}$ potential drop between electrodes as seen at the left and right sides of the figure. The spatial oscillations in the electrode potential are artifacts of the discrete ECD model and are not intended to correspond to the electric potential oscillations in a real metal. The electric potential is periodic; that is, there is not a residual electric field propagating through the system. This signifies that the electrodes do indeed behave as a Faraday cage and shield the fluid from effects of three-dimensional (3D) periodically repeated slabs of fluid as previously discussed. ${ }^{25}$ Likewise, the electric potential in the middle of the fluid is relatively flat indicating that the solution species properly shield the charges on the electrodes. 


\section{Potential of mean force}

When an ion or other species moves toward and adsorbs at the solid-liquid interface, it must pass through areas of large variations in electric field and density. The interfacial layer can be considered a free-energy barrier. This can be further quantified through calculation of the potential of mean force.

The reversible work theorem ${ }^{43}$ relates density profiles $\rho$ to the potential of mean force (PMF) $W$ :

$$
W=-R T \ln \left(\rho / \rho_{0}\right),
$$

where $\rho_{0}$ is the density at a point of reference. In this case the PMF is a measure of the free energy of adsorption or desorption. Often it is difficult to obtain the PMF using equilibration methods due to the infrequent sampling in regions of low probability. For instance, obtaining the energy barrier to surface adsorption of chloride is difficult because chloride ions rarely cross that barrier. Due to the simulation size and length, the additives do not sufficiently sample the space near the copper surface. Thus equilibrium methods are not used here to obtain the PMF.

Umbrella sampling ${ }^{44-46}$ was used to force sampling in low probability areas by imposing a harmonic potential at $x_{0}$, $U_{h}=\frac{1}{2} k_{h}\left(x-x_{0}\right)^{2}$, where $k_{h}=150 \mathrm{~kJ} / \mathrm{mol}$ and $x$ is the position relative to the metal surface. When approaching the sawtooth surface, an additional harmonic potential of constant $k_{h} / 2$ was used to fix the position in the $y$ direction above the lightly shaded copper atoms in Fig. 12. The species of interest was moved toward and away from the surface by discretely and sequentially changing $x_{o}$. The average force, $\langle f\rangle$ $=-k_{h}\left(\langle x\rangle-x_{0}\right)$, was calculated from the average position for each $x_{0}$ sampling. The average force is then integrated to give an integrated mean force (IMF) for either adsorption and desorption.

Any differences between the adsorption and desorption energies indicate an irreversible path. In order to force the solute molecule to move in a reversible adsorption path, a very detailed understanding is required. Our procedure makes the reasonable assumption that the adsorption path is largely controlled by $x$, the additive distance from the surface. However, because of the collective structure of the overall fluid and the hydration of the solute molecule, the reaction coordinate for adsorption could be more complex. Path sampling techniques such as the climbing-image, nudged-elastic-band method of Henkelman et $a l^{46}$ can be used in such circumstances.

In order to mitigate the observed hysteresis in the adsorption/desorption profiles, we did two things. First, we used the smallest steps we could in successive $x_{0}$ values $\left(\Delta x_{0}=0.2 \AA\right)$. Second, following each step we amplifed the temperature to $596 \mathrm{~K}$ for the molecules within a spherical volume ( $r=12 \AA$ ) about the additive molecule for a period of $10 \mathrm{ps}$. This heating process speeds relaxation of the fluid near the solute. The elevated-temperature region was then restored to the background temperature and allowed to equilibrate for an additional 10 ps prior to 40 ps of data collection. These efforts significantly reduced the observed hys-

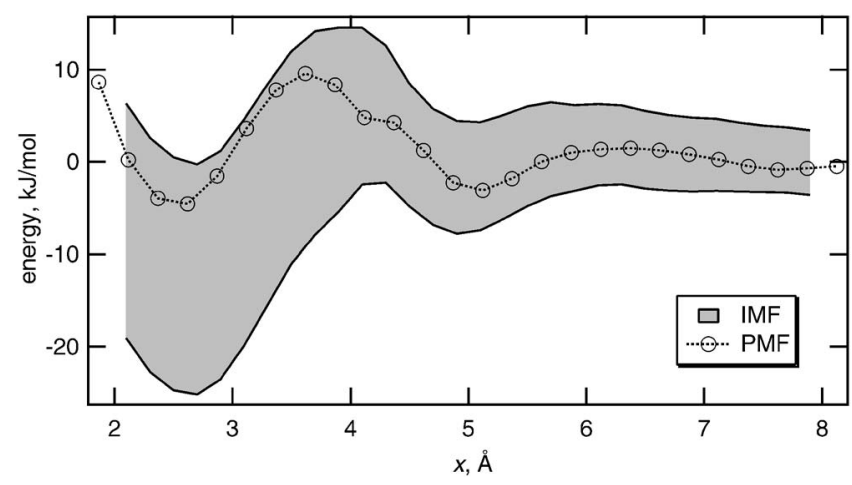

FIG. 15. Potential of mean force and integrated mean force of water at a flat copper (111) surface in the presence of sodium, chloride, copper, and bisulfate ions. The $95 \%$ confidence interval for the IMF is shown in gray. The line with circles indicates the PMF.

teresis. A significantly longer simulation time would be required to eliminate completely the hysteresis.

As shown in Fig. 15, we tested our procedure by calculating the IMF and PMF of water adsorption. The $95 \%$ confidence interval for the IMF is shown in gray and the dotted line with the points represents the PMF as found from Eq. (2). The IMF and PMF agree within statistical uncertainties. There also is not a significant difference in the IMF profiles between the adsorption or desorption paths. This indicates that in this case the forced path of approach used to calculate the IMF is reversible.

\section{B. Adsorption and desorption energies}

The IMF or apparent binding energies of MPSA-like additives near the electrode surface was obtained with the harmonic biasing potential acting on the sulfur atom of either the thiol or sulfonate groups. The remainder of the sites were free to adjust and respond accordingly. We simulated three factors that may influence species binding energy: (1) the presence or absence of $\mathrm{NaCl}$, (2) roughness of the surface, and (3) chemical functionality (thiol versus sulfonate moiety).

The results for the IMF profiles are summarized in Table VI. The potential of mean force was unreliable because of inadequate sampling. The table gives adsorption energies of thiol or sulfonate moieties of $\mathrm{MPSA}^{-}$, PDT, or $\mathrm{PDSA}^{2-}$ in their forced movement toward or away from the flat or sawtooth surface, with or without $\mathrm{NaCl}$ present, over the course of a $16 \mathrm{~ns}$ simulation. In the cases when the site is forced away from the surface, the minimum in the potential well closest to the surface is reported. For thiol and sulfonate that distance is $\sim 2.7$ and $3.0 \AA$, respectively. In the routes where the site forcibly approaches the surface, the energy is reported at the same position as in the removal. In all of the cases the difference between the force profiles for adsorption and desorption remains statistically significant, with an average value of $-67 \pm 21 \mathrm{~kJ} / \mathrm{mol}$ difference.

The approximate adsorption energy of the three organic additives studied here (MPSA ${ }^{-}$, PDT, and PDSA $^{2-}$ ) averaged over all simulation is $-19 \pm 16 \mathrm{~kJ} / \mathrm{mol}$. There is not a statistically significant difference between the binding energy of the thiol versus sulfonate molecular ends. Thus the simula- 
TABLE VI. Adsorption energies $(\mathrm{kJ} / \mathrm{mol})$ of thiol or sulfonate moieties in their forced movement toward or away from the flat or sawtooth surface, with $(\mathrm{NaCl})$ or without $(\varnothing)$ sodium chloride present. Adsorption energies are reported at 2.7 and 3.0 from the surface for thiol and sulfonate, respectively. Uncertainties at the $95 \%$ confidence level are in parentheses.

\begin{tabular}{|c|c|c|c|c|c|c|c|c|}
\hline & \multicolumn{4}{|c|}{ Thiol } & \multicolumn{4}{|c|}{ Sulfonate } \\
\hline & \multicolumn{2}{|c|}{ Toward } & \multicolumn{2}{|c|}{ Away } & \multicolumn{2}{|c|}{ Toward } & \multicolumn{2}{|c|}{ Away } \\
\hline & $\mathrm{NaCl}$ & $\varnothing$ & $\mathrm{NaCl}$ & $\varnothing$ & $\mathrm{NaCl}$ & $\varnothing$ & $\mathrm{NaCl}$ & $\varnothing$ \\
\hline & \multicolumn{8}{|c|}{ Flat surface } \\
\hline MPSA $^{-}$ & $10(10)$ & $4(7)$ & $-27(12)$ & $-53(10)$ & $6(21)$ & $-9(19)$ & $-82(19)$ & $-70(18)$ \\
\hline PDT & $-14(12)$ & $-13(8)$ & $-40(15)$ & $-47(10)$ & $\ldots$ & $\ldots$ & $\ldots$ & $\ldots$ \\
\hline \multirow[t]{2}{*}{$\mathrm{PDSA}^{2-}$} & $\cdots$ & $\cdots$ & $\cdots$ & $\cdots$ & $69(18)$ & & $-75(19)$ & \\
\hline & \multicolumn{8}{|c|}{ Sawtooth surface } \\
\hline $\mathrm{MPSA}^{-}$ & $25(13)$ & $4(14)$ & $-46(16)$ & $-24(16)$ & $33(19)$ & $20(16)$ & $-51(16)$ & $-75(17)$ \\
\hline PDT & $38(18)$ & $0(12)$ & $-25(15)$ & $-35(12)$ & $\cdots$ & $\cdots$ & $\cdots$ & $\cdots$ \\
\hline $\mathrm{PDSA}^{2-}$ & $\ldots$ & $\ldots$ & $\ldots$ & $\ldots$ & $36(25)$ & & $-87(30)$ & \\
\hline
\end{tabular}

tions do not resolve which end of MPSA ${ }^{-}$prefers to adsorb at a flat or sawtooth copper surface. Experimentally, alkanethiols (such as MPSA or PDT) can form self-assembled monolayers on copper and gold surfaces, ${ }^{47,48}$ through the binding of the thiol sulfur to the surface. It may be that the reduction of thiol to a chemically bound sulfide species, a phenomenon not incorporated in this model, strengthens the thiol adsorption energy.

Comparing the binding energies of each route on the flat versus the sawtooth surface (e.g., MPSA ${ }^{-}$in the presence of $\mathrm{NaCl}$ with the thiol forced toward and away from the flat versus rough surface), no statistically significant difference in adsorption energy is observed. When adsorption occurs at the flat surface, the species may favorably interact with the surface as well as the charged fluid layer to enhance the strength of adsorption. Since at the sawtooth surface these distinct fluid layers are disrupted, it may be expected that the adsorption energy would be reduced relative to a flat surface. It may also be that the ions occupy more of the sawtooth surface sites and thus block the adsorption of the organic species. The resolution of the IMF potential profiles is not able to differentiate between these effects.

Despite the wide variations seen in the reported energies in Table VI, a statistically significant effect of $\mathrm{NaCl}$ presence on the adsorption energy of the additives is present. At a 95\% confidence level, the addition of chloride reduces the adsorption energy by $10 \pm 9 \mathrm{~kJ} / \mathrm{mol}$ (i.e., the additive is less tightly bound to the surface) for MPSA, PDT, or PDSA. This average was generated by comparing energy differences for adsorption routes separately from desorption routes for each species, and then averaging the results. This result suggests a mechanism by which chloride reduces the copper deposition rate, namely, by displacing organic additives that would otherwise adsorb on the surface. In addition to steric hindrance on the surface, the competitive adsorption could have a Coulombic character because of the repulsion between surfacebound chloride (Fig. 13) and the negatively charged thiol and sulfonate groups of the additive molecules.

\section{Simulated electrode work function}

In the electrode charge dynamics routine, the surface charges dynamically adjust to equilibrate the chemical potential of valence charge amongst metal atoms. The work function is equivalent to this chemical potential and is a measure of the amount of work required to remove an electron from the metal out to a vacuum. The work function depends on the metal's environment. Changes in the work function are easier to compute reliably than changes in the simulated adsorption/desorption energies because the electrostatic potential of the metal fluctuates less than the forces on individual solute molecules.

The electrostatic potential of the metal can be referenced to the standard hydrogen electrode or a saturated mercuroussulfate reference electrode (SSE) by subtracting the work function of the reference electrode from the simulated work function. We approximate the work function of the standard hydrogen electrode to be $4.6 \mathrm{eV}$ (Refs. 49 and 50) (or $4.0 \mathrm{eV}$ for SSE). We offset the potential of the two simulated metal electrodes by $3.6 \mathrm{~V}$. This $3.6 \mathrm{~V}$ offset was obtained empirically in order for the simulated negative electrode to be at a potential encountered experimentally during electrodeposition.

Shown in Fig. 16 is the electrostatic potential of the simulated working electrode relative to the SSE reference for a number of perturbed cases. (The work function can be obtained by changing the ordinate units from $\mathrm{V}$ to $\mathrm{eV}$ and adding $4.0 \mathrm{eV}$ ). Upon creation of an interface (with $\sim 70 \AA$ separating the two metal surfaces), the work required to remove an electron is reduced. This step change in the work function is labeled as transition A in Fig. 16. The work required to remove an electron from the sawtooth surface is less than the flat due to the increase in exposed copper atoms resulting in an increase in the sawtooth surface energy.

When an aqueous electrolyte containing copper and bisulfate ions is introduced to fill the vacuum separating the electrode halves (transition B in the figure), the fluid should stabilize the charge on the electrode thereby increasing the work function, making it more difficult for electrons to be 


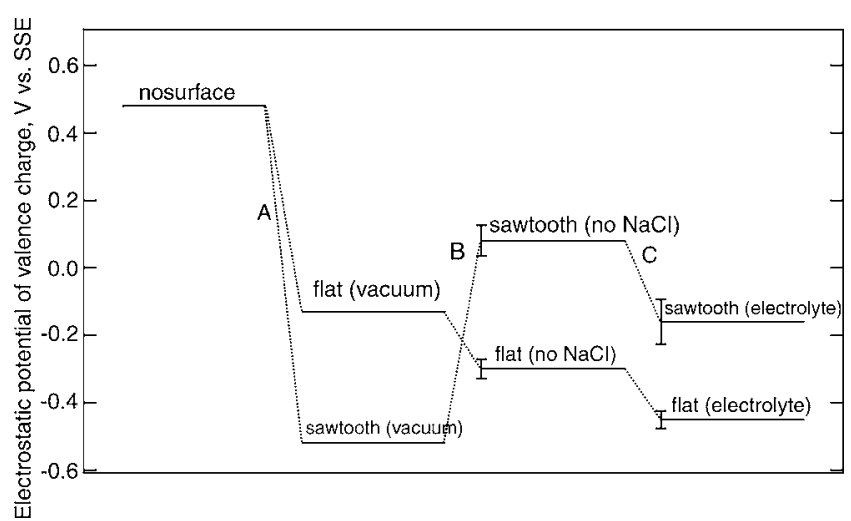

FIG. 16. Electrostatic potential of the negative electrode relative to the SSE reference when the metal interface is perturbed. Error bars represent the $95 \%$ confidence level about the mean value. The work function can be obtained by merely adding $4.0 \mathrm{~V}$ to the values found at the ordinate. The labels $\mathrm{A}, \mathrm{B}$, and $\mathrm{C}$ indicate transitions discussed in the text.

removed from the metal. Addition of electrolyte reduces the work function in the case of the flat electrode, which is not an intuitive result. It may be that the water orientation at the flat surface is such that long-range order in the fluid (evidenced by two well-defined layers of increased density) can be established and thus decrease the electrode work function due to the dipole orientation in the two water layers. Those regions of well-defined dipolar layers do not exist near the sawtooth surface.

When sodium chloride is added to the electrolyte (transition $\mathrm{C}$ in Fig. 16) the simulated work function is reduced (relative to the solution lacking sodium chloride) for both the flat and sawtooth surface geometries. This result indicates that sodium chloride causes a statistically significant increase in the ease of surface electron removal. Figure 13 shows that copper ions adsorb at least as close or even closer to the surface than does chloride. Nevertheless, chloride adsorbs closer than sodium ions. The decrease in the electrode work function upon $\mathrm{NaCl}$ addition tells us that the net effect of the additional ions is an increase in the energy of the negatively charged surface. Experimentally, it is observed that with chloride addition, the rate of copper reduction increases. A reduction in the surface work function can explain how chloride, in the absence of organic additives, increases the copper deposition rate.

A statistically significant difference in the simulated electrostatic metal potentials is not observed when adding the organic additives. The reason for this is likely due to the relatively small surface area the organic additive occupies when adsorbed at or near the surface. The species that influence the metal work function most are those that are closest to and most abundant at the surface.

Experimentally, the open circuit potential for a copper electrode in an electrolyte comprised of $0.24 M \mathrm{CuSO}_{4}$ and $1.8 \mathrm{M} \mathrm{H}_{2} \mathrm{SO}_{4}$ is $-0.41 \mathrm{~V}$ versus SSE. As shown on the right side of Fig. 16, the simulations reproduce this value quite well for electrolyte next to a flat (111) copper surface. Moreover, the ECD model of the bulk copper electrode (left side of Fig. 16) gives a work function value of about $4.5 \mathrm{eV}$ which agrees with the experimental work function for most common metals.

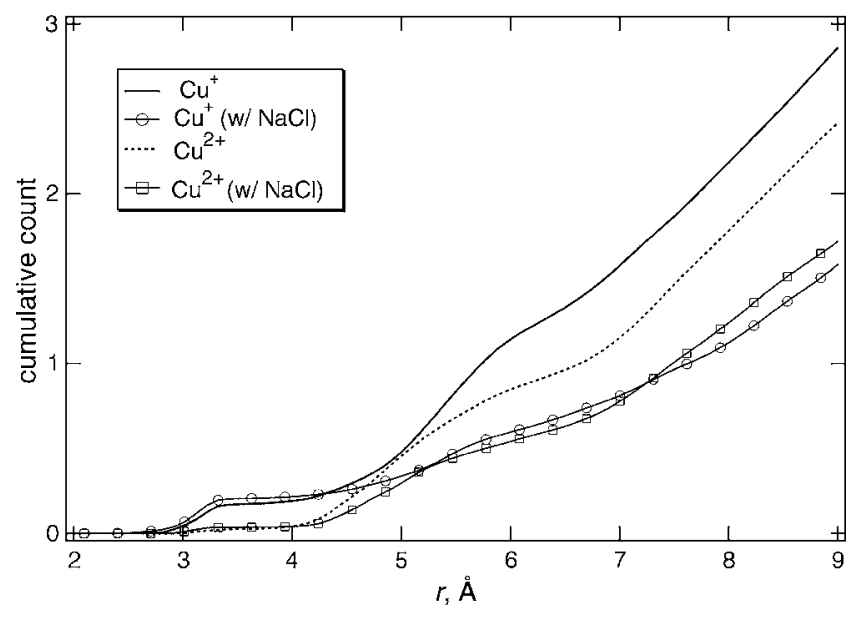

FIG. 17. Cumulative distribution of copper ions about the sulfonate group of $\mathrm{MPSA}^{-}$as a function of distance. The respective curves for the copper ions indicate baths with and without $\mathrm{NaCl}$.

\section{Copper-ion distributions about MPSA}

Experimentally it has been shown that MPSA accelerates copper deposition in the presence of chloride ions. The acceleration is greater than that which occurs when $\mathrm{NaCl}$ is added to a MPSA-free bath. ${ }^{5,8}$ The simulation results help elucidate a possible mechanism for this acceleration.

As the fluid structure near MPSA ${ }^{-}$is known in the simulations, we can obtain the distribution of cupric or cuprous ions about the thiol or sulfonate groups of $\mathrm{MPSA}^{-}$. The effects of $\mathrm{NaCl}$ on that distribution can also be resolved. The simulated structure of MPSA and the surrounding fluid can be related to the MPSA behavior near the experimental copper surface.

Figure 17 shows the distribution of copper ions around the sulfonate group of MPSA ${ }^{-}$. The curves indicate the cumulative count of ions about the sulfur atom within the spherical radius given by the abscissa. Data were collected for $\mathrm{MPSA}^{-}$that was adsorbed on or associated with a (111) copper surface (similar curves were produced for the sawtooth surface).

The most significant effect seen in Fig. 17 is that the addition of chloride ions to the bath leads to fewer cupric and cuprous ions near MPSA ${ }^{-}$. This affects both endgroups of MPSA but more particularly sulfonate. This result is consistent with recent experimental work suggesting that a complex of $\mathrm{Cl}^{-}, \mathrm{Cu}^{+}$, and sulfonate is important in accelerating copper deposition; ${ }^{4}$ another study suggested that $\mathrm{Cu}^{2+}$ is involved in the complex. ${ }^{5}$ In our simulations $\mathrm{NaCl}$ attenuates the binding energy of copper atoms to sulfonate. This attenuation of the species binding energy could lead to greater shuttling of copper ions to the surface This phenomenon can be likened to the Sabatier principle in heterogeneous catalysis, namely, that the highest turnover rate occurs when a reactive species binds only moderately to the surface.

Figure 17 also shows that cuprous ions are more prevalent than cupric ions in the immediate vicinity of the sulfonate (or thiol, not shown) sulfur. Again, this underlines the role of the solvent as we would otherwise expect that the 
more positively charged $\mathrm{Cu}^{2+}$ species would displace $\mathrm{Cu}^{+}$ next to negatively charged oxygen atoms of the sulfonate group.

\section{CONCLUSIONS}

The above sections give the intermolecular potential parameters for the simulated molecules interacting within the solution and also describe the $a b$ initio potential energy scans of copper ions, bisulfate and sulfate ions, and MPSA ${ }^{-}$approaching a $\mathrm{Cu}_{10}$ metal cluster. The ab initio data can be satisfactorily fitted with a small set of intermolecular potential parameters (Table V) within the ECD framework. The qualitative strength of adsorption at a neutral $\mathrm{Cu}_{10}$ cluster is $\mathrm{Cu}^{+}>\mathrm{Cl}^{-}>\mathrm{Na}^{+}>\mathrm{HSO}_{4}^{-}>\mathrm{MPSA}^{-}>\mathrm{H}_{2} \mathrm{O}$. At the copper surface in solution, negatively charged at a potential similar to experiment, the adsorption strength of MPSA ${ }^{-}$and $\mathrm{H}_{2} \mathrm{O}$ is reduced and follows the same order as the ab initio results.

The water structure at the interface plays a critical role in determining interfacial properties. From the species density profiles at a flat interface, chloride adsorbs both at positive and negative surfaces. High frequency oscillating layers of net negative and positive charges exist at the surface. To obtain accurate species profiles immediately in the vicinity of the solid-liquid interface, the solvent molecules must be explicitly included.

When sodium chloride is included in the simulated electrolyte, the adsorption energy of $\mathrm{MPSA}^{-}$or other like additive $(-19 \pm 16 \mathrm{~kJ} / \mathrm{mol})$ at a flat or sawtooth surface is reduced by approximately $10 \mathrm{~kJ} / \mathrm{mol}$. Sodium chloride addition also attenuates the sulfonate-copper-ion interaction and causes a decrease in the electrode work function. Such a decrease in the work function signifies that electrons can more easily move from the metal to reacting species at the surface.

This simulation work corresponds closely with a companion experimental study. ${ }^{8}$ Based on our theoretical and experimental results, proposed surface functions for chloride and the thiol and sulfonate groups in an electrodeposition bath are fourfold: (1) the thiol group causes MPSA to adhere to (or stay in the vicinity of) the metal surface; (2) the sulfonate group attracts copper ions and facilitates ion transfer to the surface, (3) $\mathrm{Cl}^{-}$attenuates MPSA adhesion and attraction of copper-ions to MPSA, and (4) $\mathrm{Cl}^{-}$reduces the surface potential.

\section{ACKNOWLEDGMENTS}

We thank Professor Tapani Pakkanen and his students at the University of Joensuu, Finland, for providing preliminary results from $\mathrm{Cu}^{+}-\mathrm{Cu}_{10}$ ab initio calculations. This work was partially supported by the donors to the ACS Petroleum Research Fund and by the National Science Foundation (No. CTS-0215786).

${ }^{1}$ T. Moffat, D. Wheeler, M. Edelstein, and D. Josell, IBM J. Res. Dev. 49, 19 (2005).

${ }^{2}$ A. C. West, J. Electrochem. Soc. 147, 227 (2000).

${ }^{3}$ P. Andricacos, C. Uzoh, J. Dukovic, J. Horkans, and H. Deligianni, IBM J. Res. Dev. 42, 567 (1998).
${ }^{4}$ Z. D. Schultz, Z. V. Feng, M. E. Biggin, and A. Gewirth, J. Electrochem. Soc. 153, C97 (2006).

${ }^{5}$ W.-P. Dow, H.-S. Huang, M.-Y. Yen, and H.-H. Chen, J. Electrochem. Soc. 152, C77 (2005).

${ }^{6}$ T. Moffat, D. Wheeler, and D. Josell, J. Electrochem. Soc. 151, C262 (2004).

${ }^{7}$ M. Tan, Ph.D. thesis, Brigham Young University, 2004.

${ }^{8}$ M. Tan, C. G. Guymon, D. R. Wheeler, and J. N. Harb, J. Electrochem. Soc. 154, D78 (2007).

${ }^{9}$ E. Spohr, Electrochim. Acta 49, 23 (2003).

${ }^{10}$ S. Izvekov and G. A. Voth, J. Chem. Phys. 115, 7196 (2001).

${ }^{11}$ I.-C. Yeh and M. L. Berkowitz, Chem. Phys. Lett. 301, 81 (1999).

${ }^{12}$ M. R. Philpott and J. N. Glosli, ACS Symp. Ser. 656, 11 (1997).

${ }^{13}$ F. Grun, M. Jardat, P. Turq, and C. Amatore, J. Chem. Phys. 120, 9648 (2004).

${ }^{14}$ GAUSSIAN98, Rev. A.6. Gaussian, Inc. Pittsburgh, PA, 1998.

${ }^{15}$ M. Dolg, U. Wedig, H. Stoll, and H. Preuss, J. Chem. Phys. 86, 866 (1987).

${ }^{16}$ H. Ruuska, R. L. Rowley, and T. A. Pakkanen, J. Phys. Chem. B 108, 2614 (2004).

${ }^{17}$ M. Frisch, J. D. Bene, J. Binkley, and H. F. Schaefer III, J. Chem. Phys. 84, 2279 (1986).

${ }^{18}$ D. R. Wheeler and R. L. Rowley, Mol. Phys. 94, 555 (1998).

${ }^{19}$ H. Berendsen, J. Grigera, and T. Straatsma, J. Phys. Chem. 91, 6269 (1987).

${ }^{20}$ D. R. Wheeler and J. Newman, J. Phys. Chem. B 108(47), 18353 (2004).

${ }^{21}$ J. L. Fulton, M. M. Hoffmann, J. G. Darab, and B. J. Palmer, J. Phys. Chem. A 104, 11651 (2000)

${ }^{22}$ A. Vishnyakov and A. V. Neimark, J. Phys. Chem. B 105, 7830 (2001).

${ }^{23}$ G. A. Kaminski, R. A. Friesner, J. Tirado-Rives, and W. L. Jorgensen, J. Phys. Chem. B 105, 6474 (2001).

${ }^{24}$ W. L. Jorgensen, J. D. Madura, and C. J. Swenson, J. Am. Chem. Soc. 106, 6638 (1984).

${ }^{25}$ C. G. Guymon, R. L. Rowley, J. N. Harb, and D. R. Wheeler, Condens. Matter Phys. 8, 335 (2005).

${ }^{26}$ T. A. Pakkanen, personal correspondence, 2004.

${ }^{27}$ G. Halász, A. Vibók, S. Suhai, and I. Mayer, Int. J. Quantum Chem. 89, 190 (2002).

${ }^{28}$ A. Ignaczak and J. Gomes, J. Electroanal. Chem. 420, 71 (1997).

${ }^{29}$ A. Ignaczak J. Gomes, and S. Romanowski, J. Electroanal. Chem. 450, 175 (1998).

${ }^{30}$ R. R. Nazmutdinov and M. Shapnik, Electrochim. Acta 41, 2253 (1996).

${ }^{31}$ A. Ignaczak, J. Electroanal. Chem. 495, 160 (2001).

${ }^{32}$ A. Ignaczak and J. Gomes, J. Electroanal. Chem. 420, 209 (1997).

${ }^{33}$ R. Hockney and J. Eastwood, Computer Simulation Using Particles (Institute of Physics, Bristol, 1988).

${ }^{34}$ M. Deserno and C. Holm, J. Chem. Phys. 109, 7678 (1998).

${ }^{35}$ M. Deserno and C. Holm, J. Chem. Phys. 109, 7694 (1998).

${ }^{36} \mathrm{~J}$. White and H. Abruna, J. Electroanal. Chem. Interfacial Electrochem. 300, 521 (1991).

${ }^{37}$ M. Tan and J. N. Harb, J. Electrochem. Soc. 150, C420 (2003).

${ }^{38}$ R. Ludwig, Angew. Chem., Int. Ed. 42, 3458 (2003).

${ }^{39}$ M. F. Reedijk, J. Arsic, F. F. A. Hollander, S. A. deVries, and E. Vlieg, Phys. Rev. Lett. 90, 066103 (2003).

${ }^{40}$ K. Doblhofer, S. Wasle, D. M. Soares, K. G. Weil, and G. Ertl, J. Electrochem. Soc. 150, C657 (2003).

${ }^{41}$ C. G. Guymon, M. L. Hunsaker, J. N. Harb, D. Henderson, and R. Rowley, J. Chem. Phys. 118, 10195 (2003).

${ }^{42}$ P. S. Crozier, R. L. Rowley, and D. Henderson, J. Chem. Phys. 113, 9202 (2000).

${ }^{43}$ D. Chandler, Introduction to Modern Statistical Mechanics (Oxford University Press, Oxford, 1987).

${ }^{44}$ D. Frenkel and B. Smit, Understanding Molecular Simulation From Algorithms to Applications (Academic, New York, 2002).

${ }^{45}$ B. Roux, Comput. Phys. Commun. 91, 275 (1995).

${ }^{46}$ G. Henkelman, B. Uberuaga, and H. Jonsson, J. Chem. Phys. 113, 9901 (2000).

${ }^{47}$ T. Ang, T. Wee, and W. Chin, J. Phys. Chem. B 108, 11001 (2004).

${ }^{48}$ C. Bain, E. Troughton, Y.-T. Tao, J. Evall, G. Whitesides, and R. Nuzzo, J. Am. Chem. Soc. 111, 321 (1989).

${ }^{49}$ J. O. Bockris and S. Khan, Surface Electrochemistry (Plenum, New York, 1993).

${ }^{50}$ A. B. Anderson and T. V. Albu, J. Am. Chem. Soc. 121, 11855 (1999). 